\title{
Seasonal Influence on Testicular Function of Male Raccoons, Procyon lotor
}

\author{
Kazuyuki KANEKO ${ }^{1)}$, Yuumi AKIYA ${ }^{1)}$, Haruno SATO ${ }^{1)}$, Ayako TANAKA ${ }^{1)}$, \\ Hajime $\mathrm{AOKI}^{2)}$, Masakazu MIYOSHI ${ }^{2)}$, Takahide ABUKAWA ${ }^{2)}$, \\ Makoto MOCHIZUKI') and Shizuo KAWAKAMI')
}

${ }^{1)}$ Department of Veterinary Obstetrics and Gynecology, School of Veterinary Medicine, Azabu University, Fuchinobe, Sagamihara, Kanagawa 229-8501, ${ }^{2)}$ Veterinary Clinical Center, Minamisorachi AMAA, Naganuma, Yubari, Hokkaido 069-1314, Japan

\begin{abstract}
In order to clarify the breeding capability of male raccoons in Japan, the testes of raccoons, a nuisance animal, collected in Kanagawa Prefecture and Hokkaido were histologically inspected. Furthermore, testosterone concentrations in their blood were measured. The testosterone concentrations increased in winter and the diameter of seminiferous tubules and the spermatogenetic score decreased in summer for the animals captured in Kanagawa Prefecture. For the animals captured in Hokkaido, the diameter of seminiferous tubules did not change and the decrease of the spermatogenetic score in summer was slight. As the above results show, there is a breeding season in male raccoons in Japan, and the reduction of testicular function in summer was greater in animals captured in Kanagawa Prefecture than in animals captured in Hokkaido.

Key words: Procyon lotor, Seminiferous tubule, Spermatogenesis, Testosterone
\end{abstract}

(J. Reprod. Dev. 51: 477-482, 2005)

$\mathbf{T}^{\mathrm{h}}$ he raccoon (Procyon lotor) is native to North America [1]. Although many have been imported to Japan as pets, many are abandoned because of their temperament. The raccoon has established itself in Kanagawa Prefecture and westcentral Hokkaido and has been causing large social problems by damaging agricultural products and getting into mischief. Although an eradication program in which feral raccoons were trapped had been carried out, it did not solved the problem because of the raccoons' high reproductivity, so a fundamental study to measure effective extermination of the animal is required [2]. One measure of solving this problem might be to control

Accepted for publication: April 19, 2005

Published online: June 10, 2005

Correspondence: K. Kaneko (e-mail: kaneko@azabu-u.ac.jp) the breeding capability of the male [1]. However, the male breeding cycle is not well known [3]. The lengthening of days and rise of temperatures serve as triggers for the breeding season [4], with the breeding season in the U.S. being January and February [3]. The copulation peak in Hokkaido is also in February [5], and most litters are born in April. However, in Kanagawa Prefecture, newborns have even been captured in autumn. There are also individual animals that give birth between August and September in the U.S. [1, 4]. In this study, the testes of raccoons were histologically examined and the concentration of testosterone in serum was measured in order to investigate seasonal changes in testicular function in male raccoons captured in Kanagawa Prefecture and Hokkaido. 


\section{Materials and Methods}

\section{Animals}

Male raccoons that had been a nuisance in Kanagawa Prefecture and Hokkaido were captured between October 2001 and October 2003. When the testes did not descend in to the scrotum or the penis was nonextrusible, the animals were considered juveniles or yearlings [6], and were excluded. The number of individual animals used each month was 2 in January, 2 in February, 5 in March, 2 in April, 2 in May, 1 in June, 0 in July, 2 in August, 4 in September, 4 in October, 1 in November, and 3 in December for Kanagawa Prefecture, and 6 in June, 2 in July, 2 in August, 3 in September, and 1 in October for Hokkaido. After being captured, 288 mg of ketamine hydrochloride (Ketalar 50, Sankyo Co. Ltd., Tokyo, Japan) was given intramuscularly to each individual animals as a sedative, body weight was measured, and both testes were extracted for historical examination. Blood was collected by puncturing the raccoon's heart, and was allowed to clot at room temperature. After centrifugation, the serum was removed, frozen at $-18 \mathrm{C}$, and stored until hormone assay was performed.

\section{Histology}

The testes were fixed in $10 \%(\mathrm{v} / \mathrm{v})$ formaldehyde, embedded in paraplast, separated into $4 \mu \mathrm{m}$ sections, and stained with hematoxylin and eosin. Seminiferous tubule diameters were measured in micrometer, and the mean value of 20 tubules for each testis was calculated. Spermatogenesis evaluation was principally based on the most advanced spermatogenetic cells. The score count criteria were as follows: Score 1: spermatogonia only; Score 2: no cells further than primary spermatocytes; Score 3: some cells further than secondary spermatocytes; Score 4: some cells further than spermatids; Score 5: spermatozoa. In most cases, the whole sample was examined under a light microscope using low $(\times 100)$ and then high $(\times 400)$ magnification.

\section{Radioimmunoassay}

Testosterone concentrations were measured by radioimmunoassay (RIA) using kits purchased from Diagnostic Products Corp. (Los Angeles, CA, USA). All procedures were conducted according to the manufacturer's instructions. The intra-assay and inter-assay coefficients of variation and calibration range of these assays were $<11.2 \%$ and $<11.8 \%$ and $4-1,600 \mathrm{ng} / \mathrm{ml}$, respectively.

\section{Statistical analysis}

Because the number of animals captured each month was small, the data was compared among the four seasons as follows: winter (January to March), spring (April to June), summer (July to September), and fall (October to December). The diameter of the seminiferous tubules, spermatogenetic scores, and serum concentrations of testosterone were analyzed using ANOVA followed by Fisher's protected least-significant difference post-hoc analysis. The thresholds used to detect significant differences were $5 \%$ or $1 \%$.

\section{Results}

The average values for the seminiferous tubule diameter by season are shown in Fig. 1. For the animals captured in Kanagawa Prefecture, the value peaked in winter $(261.3 \pm 17.3 \mu \mathrm{m})$ and slightly decreased in spring. In summer, it fell rapidly and showed a minimum value $(177.2 \pm 11.0$ $\mu \mathrm{m})$. Subsequently, it began to increase in fall, and returned to the same value as found in spring. There were significant differences between summer, winter, spring, and fall $(\mathrm{P}<0.01)$. For the animals captured in Hokkaido, the seminiferous tubule diameter did not change and there were no large reductions in summer as observed in Kanagawa Prefecture.

The average values for the spermatogenetic scores by season are shown in Fig. 2. For the animals captured in Kanagawa Prefecture, spermatogenetic scores showed similar changes as observed in the diameter of seminiferous tubules. It was maintained from winter to spring, and spermatids or spermatozoa were observed in all samples (Fig. 4). However, the score fell to $1.5 \pm 0.6$ in summer, and only spermatogonia were observed in many samples (Fig. 4). After that, the score began to increase again in fall, and spermatids or spermatozoa were observed again. There were significant differences between summer, winter, spring, and fall $(\mathrm{P}<0.01)$. For the animals captured in Hokkaido, the spermatogenetic score in spring was $4.6 \pm 0.2$ and spermatids or spermatozoa were observed in all samples. Although it fell to $3.9 \pm 0.6$ 


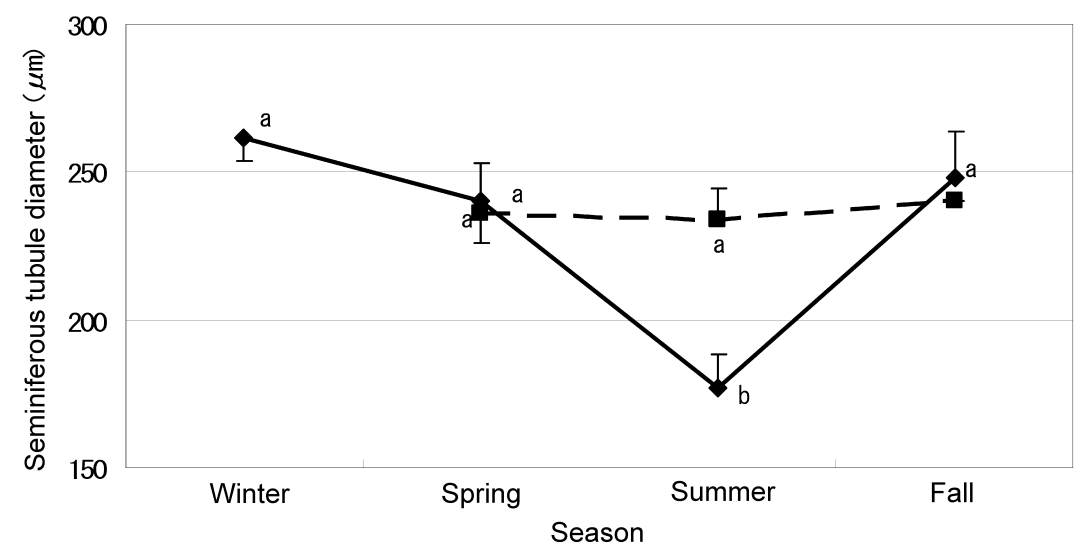

Fig. 1. Changes in seminiferous tubule diameter for animals captured in Kanagawa Prefecture $(\diamond)$ and Hokkaido $(\square)$. Values shown are the means \pm SE. ${ }^{a, b}$ The values with different superscripts differ significantly $(\mathrm{P}<0.01)$.

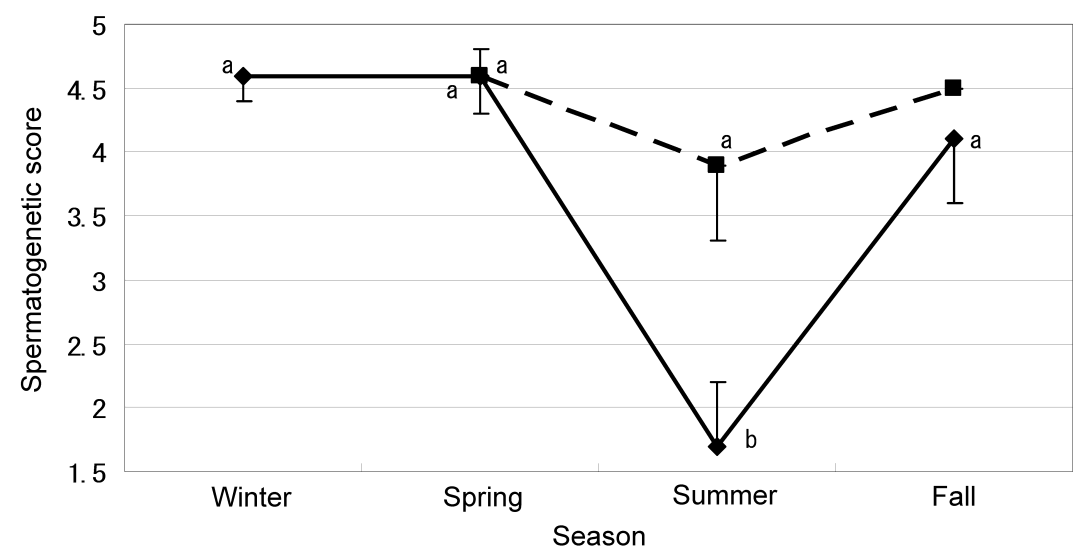

Fig. 2. Changes in spermatogenetic score for animals captured in Kanagawa Prefecture $(\diamond)$ and Hokkaido $(\square)$. Values shown are the means \pm SE. ${ }^{a, b}$ The values with different superscripts in the same group differ significantly $(\mathrm{P}<0.05)$.

in summer, there were no large reductions as observed in Kanagawa Prefecture. A significant difference was observed between Kanagawa Prefecture and Hokkaido in summer $(\mathrm{P}<0.05)$.

The averages value of testosterone concentrations by season are shown in Fig. 3. For the animals captured in Kanagawa Prefecture, testosterone concentrations did not fluctuate greatly from winter to summer, and stayed around $20 \mathrm{ng} / \mathrm{ml}$. However, it increased rapidly and reached $73.4 \pm 53.2 \mathrm{ng} / \mathrm{ml}$ in fall. For the animals captured in Hokkaido, testosterone concentrations were under $20 \mathrm{ng} / \mathrm{ml}$ in spring and summer. Although only one animal was available in fall, it showed a high testosterone level $(84 \mathrm{ng} / \mathrm{ml})$. There were no significant differences between seasons for both Kanagawa Prefecture and Hokkaido.

\section{Discussion}

Wild animals need to give birth in the most suitable season for raising their young. A wide variety of environmental cues are used to synchronize the breeding season, and the specific environmental factors that regulate reproductive activity can vary from species to species [7]. These 


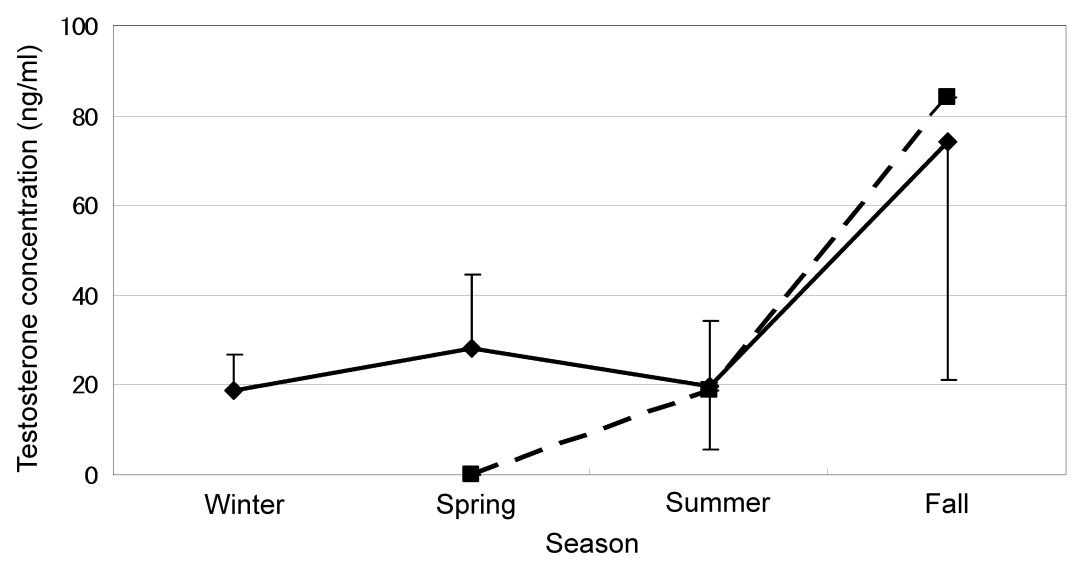

Fig. 3. Changes in blood testosterone concentration for animals captured in Kanagawa Prefecture $(\bullet)$ and Hokkaido $(\square)$. Values shown are the means \pm SE.

A

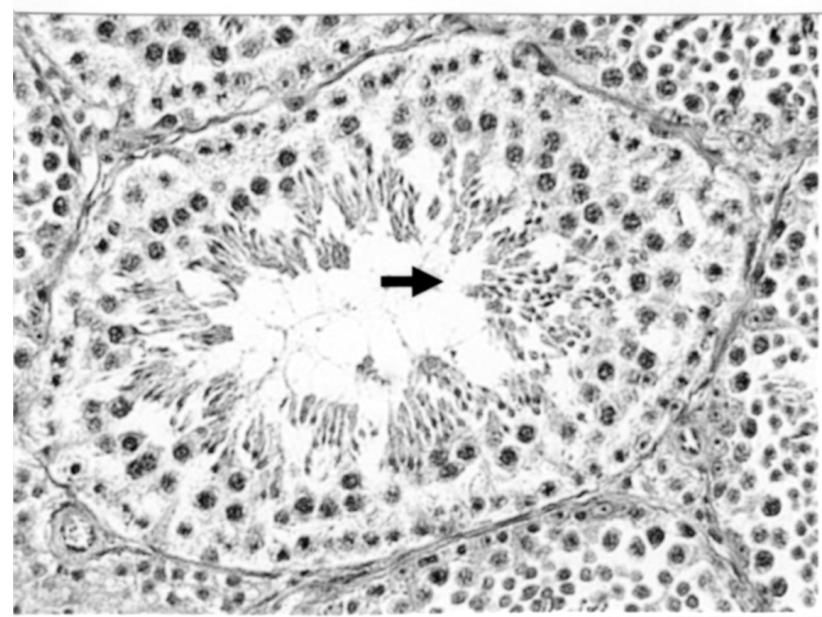

B

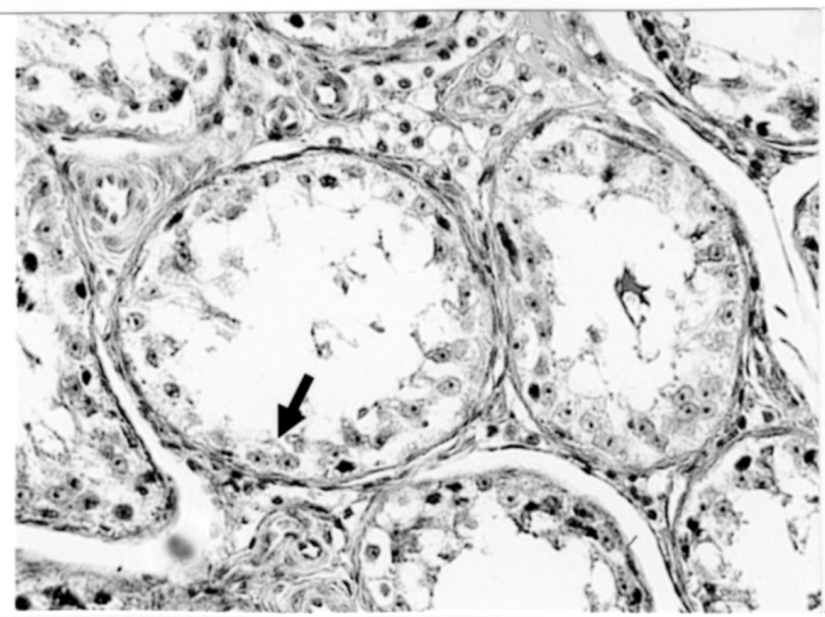

Fig. 4. Testicular sections (hematoxylin). A: A testicular section from a raccoon captured in Kanagawa Prefecture in January. The spermatogonesis is active. The arrow indicates the spermatids. B: A testicular section from a raccoon captured in Kanagawa Prefecture in August. Spermatogonesis is inactive, and only spermatogonia and primary spermatocytes are observed. The arrow indicates spermatogonia. $\times 400$.

factors include food availability, competition, predatory pressure, and climatic factors like temperature or humidity, etc. [8]. For instance, late snows and low temperatures can limit a raccoon's movement and delay mating in animals that are capable of breeding in the far Northern latitude areas of the United States [9]. Or male raccoons live separately from females after the mating period and no associations between males and females are apparent [9]. Since contacts with female animals stimulate the libido in rams [10], this separation from female raccoons might also be related to the decrease of testicular function after the mating period. However, the winter climate in Kanagawa Prefecture is relatively calm, and there are no limits on breeding activity caused by weather or large environmental changes throughout the year.

Despite the important role played by environmental factors in the timing of seasonal reproductive cycles, there is substantial evidence 
that annual reproductive cycles can be generated endogenously in the absence of any annual change in environmental factors [7]. These cycles are normally synchronized by periodic environmental signals, such as the seasonal change in day length [7]. Day length affects melatonin secretion from the pineal gland, and melatonin controls the function of the gonads [11]. Although testosterone is related to spermatogenesis, the testosterone concentration in serum of male raccoons has not been reported. In the male raccoon dog (Nyctereutes procynoides), which is supposed to have the same breeding season as raccoons, there is a clear seasonal change in testosterone concentration in serum or spermatogenetic score, with the concentration beginning to increase in fall, reaching a peak during breeding season in February, and remaining at a basic level between May and August [12, 13]. In this research, the testosterone concentration was high in fall as observed in the male raccoon dog in both Kanagawa Prefecture and Hokkaido. This fact suggests that there is a seasonal change in testicular function generated by endogenous factors. Because the latitudes of raccoon habit in the United States and Japan are almost same, between $30^{\circ}$ and $45^{\circ}$ north, the breeding season might be similar in both countries.

There were seasonal changes in the diameter of the seminiferous tubules and spermatogenetic scores. They decreased in summer and began to increase again in fall for animals captured in Kanagawa Prefecture. These seasonal changes are mostly in agreement with the existence of sperm in the epididymis as reported by Sanderson et al. [14]. They reported that the sperm presence rate in the epididymis of male raccoons captured in Illinois was almost $100 \%$ in January to May, plunging to $35 \%$ in June, becoming $0 \%$ in September, then beginning to go up again in October [14]. In this research, the time when the testosterone concentration increased corresponded to the time when the testicular function was restored in the animals captured in Kanagawa Prefecture. Accordingly, the seasonal change in testicular function may be controlled by testosterone. However, in regard to the animals captured in Hokkaido, the reduction in the spermatogenetic score was slight and there were no seasonal changes in the diameter of the seminiferous tubules. The environmental temperature also affects male testicular function. Especially in boar, lowered fertility is observed following periods of extremely hot weather, and this is known as seasonal (summer) infertility syndrome [15]. The normal temperature of Yokohama in Kanagawa Prefecture between July and September is around 25 degrees [16]. The normal temperature of Illinois in summer is similar to that of Kanagawa Prefecture. On the other hand, the summer climate of Hokkaido is relatively cool and the normal temperature is around 20 degrees [16]. This suggests that high temperatures might affect testicular function in male raccoons.

Overall, these results suggest that there might be a breeding season in male raccoons in Japan, and the reduction of testicular function in summer was greater in the animals captured in Kanagawa Prefecture than in the animals captured in Hokkaido.

\section{Acknowledgements}

We would like to thank Dr. Takashi Makino of the Kanagawa Nature Protection Center for his help in collecting samples.

\section{References}

1. Rosatte RC. Management of raccoons (Procyon lotor) in Ontario, Canada: do human intervention and disease have significant impact on raccoon population? Mammalia 2000; 64: 369-390.

2. Hokkaido Government. White Book on Environmental in Hokkaido. Hokkaido Environmental Policy Planning Div, Sapporo 2001 (in Japanese).

3. Stuewer FW. Reproduction of raccoons in Michigan. J Wildl Manage 1943; 7: 60-73.
4. Lotze J-H, Anderson S. Procyon lotor. Mammalian Species 1979; 119: 1-8.

5. Asano M, Matoba Y, Ikeda T, Suzuki M, Asakawa M, Ohtaishi N. Reproductive characteristic of the feral raccoon (Procyon lotor) in Hokkaido, Japan. J Vet Med Sci 2003; 65: 369-373.

6. Nalbandov AV. Reproductive Physiology. San Francisco: W. H. Freeman and Company; 1958: 271.

7. Turek FW, Cauter EV. Rhythms in reproduction. In: Knobil E, Neill JD (eds.), The Physiology of 
Reproduction. New York: Raven Press; 1988: 1789 1830.

8. Bronson FH. Seasonal regulation of reproduction in mammals. In: Knobil E, Neill JD (eds.), The Physiology of Reproduction. New York: Raven Press; 1988: 1831-1871.

9. Zeveloff SI. Social organization. In: Bolen EA (eds.), Raccoons, A Natural History. Washington and London: Smithsonian Institution Press; 2002.

10. Holmes RJ. Sexual behavior of Seep. In: Morrow DA (ed.), Current Therapy in Theriogenology. 2nd ed, Philadelphia: WB Saunders Company; 1987: 870-873.

11. Sharp DC. The effects of photoperiod on reproduction in the mare and methods of artificial control. In: Morrow DA (ed.), Current Therapy in Theriogenology. 2nd ed, Philadelphia: WB Saunders Company; 1987: 665-670.

12. Qiang W, Murase T, Tsubota T. Seasonal changes in spermatogenesis and testicular steroidgenesis in wild male raccoon dogs (Nyctereutes procynoides). J Vet Med Sci 2003; 65: 1087-1092.

13. Yongjun $X$, Shuxian J, Yuren T, Jianhua S, Guosen $\mathbf{W}$. Seasonal changes in testicular size and serum testosterone levels and the relationship between serum testosterone concentration and reproductive performance in male raccoon dogs. Scientifur 1994; 18: 39-43.

14. Sanderson GC, Nalbandov AV. The reproductive cycle of the raccoon in Illinois. Ill Nat Hist Surv Bull 1973; 31: 29-85.

15. Crabo BG. Factors affecting spermatogenesis and boar fertility. In: Morrow A (ed), Current Therapy in Theriogenology 2. Philadelphia: W. B. Saunders Company 1986; 975-978.

16. The Japanese Meteorological Agency. Climate in the 20th Century of Japan. Tokyo: The Printing Bureau, Finance, 2002; 110-111. 